\title{
Atenção odontológica e práticas de higiene bucal em instituições de longa permanência geriátricas
}

\author{
Dental care and oral hygiene practices \\ in long-term geriatric care institutions
}

Raquel Conceição Ferreira ${ }^{1}$

Carolina Wolff Schwambach ${ }^{2}$

Cláudia Silami de M agalhães ${ }^{2}$

Allyson N ogueira M oreira ${ }^{2}$

\footnotetext{
${ }^{1}$ Departamento de Odontologia, Centro de Ciências Biológicas e da Saúde, Universidade Estadual de M ontes Claros. Campus Universitário Professor Darcy Ribeiro, $\mathrm{s} / \mathrm{n}^{\circ}$, Vila M auriceia. 39401-089 Montes Claros MG.

ferreira_rc@hotmail.com ${ }^{2}$ Departamento de

Odontologia Restauradora, Faculdadede O dontologia, UniversidadeFederal de M inas Gerais.
}

Abstract This study evaluated the activities of dentists, dental care and oral hygiene practices in the long-term care institutions of Belo H orizonte (M inas Gerais, Brazil). A semi-structured questionnaire was handed out to the coordinators of 37 philanthropic and 30 private institutions. The data was compared by the chi-square and Fisher's Exact Tests. 81\% of the questionnaires were answered. The majority of the private ( $74.2 \%)$ and philanthropic institutions ( $87 \%$ ) do not have a dentist $(p=0.21)$. The location, period of existence, type institution kind and number of residents weren't factors regarding the presence of a dentist $(p>0.05) .67 \%$ of the philanthropic institutions with equipped consultation rooms had dentists, though there were none when there was no consultation room. Even without consultation rooms, $13 \%$ of the private institutions had dentists. When necessary, $69.6 \%$ of the philanthropic institutions refer the elderly to public health centers, while $58.1 \%$ of the private institutions refer them to their family dentists. A higher percentage of the private institutionsadopted systematic oral hygi ene procedures $(p=0.01)$, with a considerable divergence of treatment reported. There is a need to include a dentist on the health staff in the institutions and for systematization of oral hygiene practices.

Key words Integrated healthcare for the aged, Oral hygiene, Long-term care institutions for the elderly
Resumo Este estudo avaliou a atuação de cirurgiões-dentistas (CD) nasinstituições de longa permanência de Belo H orizonte (M G) e as práticas de higiene bucal adotadas. Q uestionários semiestruturados foram entregues aos coordenadores das 37 instituições filantrópicas e trinta privadas e coletados após uma semana. Os resultados foram comparados pelos testes Q ui- quadrado eExato de Fisher $(p<0,05)$. H ouve retorno de $81 \%$ dos questionários. A maioria das instituições privadas (74,2\%) e filantrópicas (87\%) não possui CD na equipe de saúde $(p=0,21)$. A localização da instituição, o tempo de fundação, o tipo e o número de residentes não interferiram na presença de $C D$ $(p>0,05)$. N as institui ções filantrópicas com consultório, $67 \%$ possuíam $C D$, enenhum CD trabaIhava onde não havia consultório. M esmo sem consultório, 13\% das instituições privadas possuíam CD. 69,6\% das filantrópicas encaminhavam o idoso em caso denecessidade para centros desaúdee, nas privadas, $58,1 \%$ direcionavam ao famili$\operatorname{ar}(p=0,00)$. M aior percentual de instituições privadas adotava medidas sistematizadas de higiene bucal ( $p=0,01)$, com grandevariabilidadenas condutas relatadas. Há necessidade da incorporação do CD na equipe de saúde das instituições e da sistematização das práticas de higiene bucal.

Palavras-chave A tenção integral ao idoso, Saúde bucal, Instituiç̧ões de longa permanência para idosos 
Introdução

A população brasileira vem envelhecendo de forma rápida. Paral elamente às transformações no perfil demográfico, o Brasil vem sofrendo profundas mudanças sociais como a urbanização e as alterações na estrutura familiar. Esses fatores poderão contribuir para um aumento da demanda por instituições de longa permanência $a^{1,2}$, que deverão ser parteintegranteeindispensável dosprogramas deatenção à população idosa. 0 envelhecimento populacional vem sendo motivo degrande preocupação pelas implicações que pode ter no atendimento às necessidades básicas do idoso. Tal situação atingepolíticas públicas de ação específica sobre idosos, para a promoção do seu bemestar físico, social, econômico e psicológico ${ }^{1}$.

$\mathrm{Na}$ área da saúde bucal, há uma ausência de programas voltados para os idosos e um quadro epidemiológico precário. No Levantamento Epidemiológico em Saúde Bucal realizado no Brasil pelo M inistério da Saúde de 2002 a 2003, 0 edentulismo foi considerado um grave problema no grupo etário de 65 a 74 anos $^{3}$. Outros estudos mostraram o mesmo quadro de ausência de dentes, além de alta prevalência de doença periodontal, lesões de mu cosa, al to índice de uso e necessidade de próteses ${ }^{4-7}$. Segundo Silva eValsecki Jr. ${ }^{5}$, os idosos institucionalizados, devido ao seu maior grau de fragilidade, apresentam pior condição de saúde bucal do que os idosos não institucionalizados. A população idosa nas instituições é muito heterogênea quanto às necessidades e habilidade de autocuidado, possuindo um número acentuado de pessoas mental e fisicamente fragilizadas 8 .

Entre os cuidados de saúde de responsabilidade das instituições, estão incluídos os cuidadosodontológicos 9 . Entretanto, a negligênciacom os cuidados bucais em instituições tem sido documentada, e diferentes explicações, como falta de tempo, conhecimento, interesse, dificuldade em lidar com idosos com comprometimento funcional e cognitivo, são dadas pelos responsáveis por esta atenção $0^{10}$. Esta situação pode contribuir para deterioração da saúde bucal dosidosos, potencializando os problemas existentes.

0 objetivo desse estudo foi comparar as instituições de longa permanência filantrópicas e privadas de Belo Horizonte quanto a variáveis sociodemográficas e à composição da equipe de saúde e avaliar a atuação de cirurgiões-dentistas nessas instituições, bem como as práticas de higiene bucal adotadas.

\section{Métodos}

Trata-se de um estudo transversal realizado nas instituições de longa permanência geriátricas de Belo H orizonte, M inas Gerais, Brasil, em fevereiro de 2006. Participaram todos os coordenadores das trinta instituições filantrópicas e 37 privadas, distribuídas nas nove regionais administrativas do município de Belo Horizonte (Leste, Oeste, Norte, Centro-Sul, Noroeste, Nordeste, Venda-N ova, Pampulha, Barreiro).

Os dados foram obtidos por meio de um questionário semiestruturado autoaplicado, previamente testado. Esse instrumento foi entregue em mãos, pelo pesquisador, aos coordenadores das instituições participantes do estudo durante o seu turno de trabalho. Após o período de uma semana, os questionários respondidos foram coletados pelo mesmo pesquisador. Previamente à entrega do questionário, foi realizado um contato tel efônico com todos os coordenadores das instituições para confirmação de endereço, esclarecimentos sobre a pesquisa e sobre a visita do pesquisador. Nesse primeiro contato, também foram coletadas informações sobre o número de idosos residentes e sua distribuição por sexo. A lista atualizada das instituições de longa permanência filantrópicas e privadas, com 0 nome dos coordenadores das instituições e o telefone para contato, foi fornecida pela Promotoria de D efesa da Pessoa Portadora de D eficiência Física e do Idoso.

As instituições de longa permanência privadas e filantrópicas foram caracterizadas quanto ao número de residentes e sua distribuição por gênero, à localização nas nove regionais administrativas de Belo Horizonte, ao tempo de fundação, ao tipo (segundo sua composição por gê nero do residente: masculina, feminina ou mista) e quanto à constituição da equipe de saúde atuante.

A variável dependente deste estudo foi a oferta de atenção odontológica avaliada pela presença de um ou mais cirurgiões- dentistas em atividade na instituição, sendo a resposta dicotômica: com ou sem oferta de atenção. As variáveis independentes foram modalidade da instituição (filantrópica ou privada), presença de consultório odontológico convencional na instituição, tipo, localização e tempo de fundação da instituição e número de idosos residentes.

Além disso, os coordenadores foram questionados quanto às medidas adotadas pelas instituições em relação à saúde bucal dos residentes e à rotina de higiene bucal dos idosos. Estas duas 
últimas questões foram avaliadas por perguntas não estruturadas.

Esteprojeto foi aprovado pelo ComitêdeÉtica em Pesquisa da Universidade Federal de Minas Gerais. Todos os coordenadores das instituições assinaram o termo de consentimento livre e esclarecido.

Os dados foram submetidos à análise descritiva. As variáveis numéricas foram comparadas pelo testeM ann-Whitney. A existência de diferenças entre as modalidades das instituições (privada efilantrópica) ea relação entre a oferta ou não deatenção odontológica nas instituições delonga permanência de Belo H orizonte com as variáveis independentes listadas foram verificadas por meio do teste Qui-quadrado de Pearson ou Exato de Fisher. 0 nível designificância considerado foi de $5,0 \%$. Para a realização dos testes, foi empregado o pacote estatístico Epi Info versão 3.3.2.

\section{Resultados}

No momento da coleta de dados, residiam 1.669 idosos nas instituições pesquisadas ( $68 \%$ nas filantrópicas), com um predomínio de mulheres $(74 \%)$, tanto nas instituições privadas $(71 \%)$ como nas filantrópicas (75\%). 0 número médio de idosos nas instituições privadas foi de 15 idosos/instituição ( $\pm 9 ; 4$ a 38) e nas filantrópicas de 38 idosos/instituição $( \pm 31 ; 7$ a 120$)(p=0,00)$. As instituições estavam heterogeneamente distribuídas no município de Belo H orizonte $(\chi 2=21,07$; $p=0,00$ ), com as instituições privadas mais frequentemente localizadas nas regionais CentroSul e Pampulha e as filantrópicas nas regionais Barreiro, Leste, N ordeste, Norte, Oeste e Venda N ova (Gráfico 1).

A taxa de resposta foi de $81 \%$ ( 54 questionários), e os dados apresentados a seguir se referem a 23 instituições filantrópicas (76,7\%) e 31 privadas $(83,8 \%)$.

As instituições filantrópicas avaliadas foram, em média, mais antigas que as privadas, apresentando maior tempo de fundação (filantrópicas $=28,17 \pm 19,14$ anos; privadas $=5,87 \pm 4,92$ anos) $(p=0,00)$.

Asinstituições filantrópicas e privadas diferiram entresi segundo o seu tipo, baseado na composição dos seus residentes por gênero $(\chi 2=$ $16,58 ; p=0,00)$, havendo entre as privadas um predomínio do tipo “mista' (93\%) e nenhuma instituição "masculina”. Entre as filantrópicas, a maioria das instituições era "feminina" (52\%), seguindo-se instituições que recebiam indivíduos de ambos os sexos (44\%).

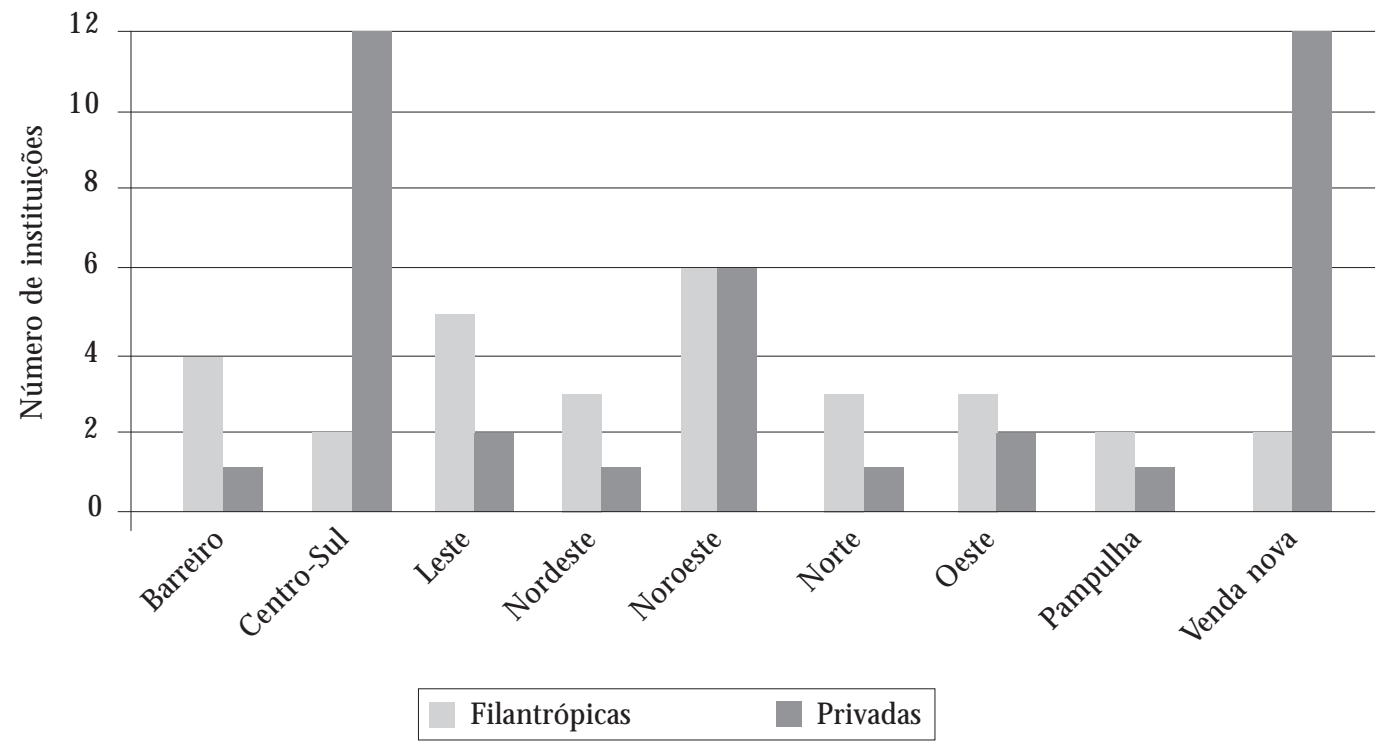

Gráfico 1. Distribuição das instituições de longa permanência filantrópicas e privadas de Belo Horizonte (MG) por regiões administrativas do município. 
$\mathrm{Na}$ análise da equipe de saúde, de maneira geral, maior percentual de instituições privadas possuía profissionais de diferentes áreas de atuação. 0 cirurgião-dentista fazia parte da equipe de saúde em $25,8 \%$ e $13 \%$ das instituições privadasefilantrópicas, respectivamente. Considerando toda a equipe de profissionais de saúde das instituições, os cirurgiões-dentistas estavam entre os que menos frequentemente apareceram (Tabela 1).

Asvariáveis avaliadas não apresentaram efeito significativo sobre a oferta de atenção odontológica nas instituições participantes. Na maioria das instituições foi observada ausência de atenção odontológica, independentemente da modalidade, da localização, do tempo de fundação, do tipo, do número de idosos residentes e da presença de consultório odontológico convencional (Tabela 2).

A relação entre a presença de consultório odontológico convencional e a atenção odontológica não foi significativa. No entanto, foi verificado que todas as três instituições que possuíam consultório odontológico eram filantrópicas. Assim, ao analisar essa relação isolando o efeito da modalidade, a presença de consultório odontológico convencional interferiu positivamente na presença deatenção odontológica nas instituições filantrópicas. Nas instituições com consultório, $67 \%$ possuíam pelo menos um cirurgião-dentista em atividade, e entre as quenão possuíam consultório, somente em uma delas ( $5 \%$ ) havia atenção odontológica $(p=0,03)$. No entanto, mesmo sem consultório odontológico convencional, foi observado que $25,8 \%$ das instituições privadas ofertavam serviços odontológicos aos seus residentes por meio da contratação de atendimento domiciliar com ou sem autilização de consultórios odontológicos portáteis (Tabela 3).

Quando os coordenadores das instituições delonga permanência foram questionadosquanto às medidas adotadas pelas instituições em relação à saú de bucal dos residentes, houve, no total dos casos, relato de encaminhamento perante uma necessidade. Na mai oria $(69,6 \%, n=16)$ das instituições filantrópicas, os coordenadores re lataram encaminhar o idoso para um centro de saúde. N as instituições privadas, a família foi responsabilizada pelo encaminhamento do idoso na maioria dos casos $(n=18 ; 58,1 \%)$. Vale ressaltar que em oito $(25,8 \%)$ instituições privadas, os coordenadores relataram contratar um cirurgião-dentista para realização de atendimento na própria instituição com o uso ou não deconsultórios odontológicos portáteis. Nas filantrópicas, esse tipo de atenção não foi citado pelos coordenadores das instituições (Gráfico 2).

Os coordenadores das instituições de longa permanência privadas $(90,3 \%)$ relataram com mais frequência que aqueles das filantrópicas (60,9\%) a adoção deuma rotina dehigiene bucal e das próteses nos idosos residentes $(p=0,01)$. As práticas de higiene bucal relatadas pelos coordenadores variaram muito entre as instituições, evidenciando a ausência de uma sistematização das condutas adotadas entre elas.

\section{Discussão}

A taxa de resposta dos questionários foi de $81 \%$, considerada "muito boa" para estudos em que esseinstrumento éempregado ${ }^{11}$. Embora os questionários tenham sido entregues pelo pesquisa-

Tabela 1. Frequência absoluta e relativa das instituições filantrópicas e privadas que possuem profissionais de saúde nas diferentes áreas de atuação. Belo H orizonte (M G), 2006.

\begin{tabular}{lcc}
\hline \multicolumn{1}{c}{ Profissionais de saúde } & Instituições privadas $(n=31)$ & Instituições filantrópicas $(n=23)$ \\
\hline Médico & $23(74,2 \%)$ & $21(91,3 \%)$ \\
Enfermeiro & $8(25,8 \%)$ & $6(26,1 \%)$ \\
Técnicos de enfermagem & $22(71 \%)$ & $11(47,8 \%)$ \\
Auxiliar de enfermagem & $24(77,4 \%)$ & $16(69,6 \%)$ \\
Psicólogo & $13(41,9 \%)$ & $11(47,8 \%)$ \\
Assistente social & $5(16,1 \%)$ & $9(39,1 \%)$ \\
Nutricionista & $25(80,6 \%)$ & $8(34,8 \%)$ \\
Cirurgião-dentista & $8(25,8 \%)$ & $3(13 \%)$ \\
Fisioterapeuta & $28(90,3 \%)$ & $15(65,2 \%)$ \\
Cuidadores formais & $25(80,6 \%)$ & $18(78,3 \%)$ \\
Terapeuta ocupacional & $25(80,6 \%)$ & $11(47,8 \%)$ \\
\hline
\end{tabular}


Tabela 2. Distribuição proporcional das instituições de longa permanência de Belo Horizonte segundo a oferta de aten ção odontológica e as variáveis independentes avaliadas. Belo Horizonte (M G), 2006.

\begin{tabular}{|c|c|c|c|}
\hline \multirow{2}{*}{ Variáveis independentes avaliadas } & \multicolumn{2}{|c|}{ Oferta de atenção odontológica } & \multirow[b]{2}{*}{ Valor de $\mathrm{p}$} \\
\hline & Sim & Não & \\
\hline \multicolumn{4}{|l|}{ Modalidade da instituição } \\
\hline Filantrópica & $3(13 \%)$ & $20(87 \%)$ & \multirow{2}{*}{$p=0,21$} \\
\hline Privada & $8(25,8 \%)$ & $23(74,2 \%)$ & \\
\hline \multicolumn{4}{|l|}{ Localização da instituição } \\
\hline Barreiro & $0(0 \%)$ & $3(100 \%)$ & \multirow{9}{*}{$p=0,50$} \\
\hline Centro-sul & $4(36,4 \%)$ & $7(63,6 \%)$ & \\
\hline Leste & $1(20 \%)$ & $4(80 \%)$ & \\
\hline Nordeste & $1(33,3 \%)$ & $2(66,7 \%)$ & \\
\hline Norte & $1(50 \%)$ & $1(50 \%)$ & \\
\hline Oeste & $1(20 \%)$ & $4(80 \%)$ & \\
\hline Pampulha & $3(25 \%)$ & $9(75 \%)$ & \\
\hline Venda Nova & $0(0 \%)$ & $2(100 \%)$ & \\
\hline Noroeste & $0(0 \%)$ & $11(100 \%)$ & \\
\hline \multicolumn{4}{|l|}{ Tempo de fundação } \\
\hline 0 a 10 anos & $6(20 \%)$ & $24(80 \%)$ & \multirow{2}{*}{$p=0,94$} \\
\hline 11 anos ou mais & $5(20,8 \%)$ & $19(79,2 \%)$ & \\
\hline \multicolumn{4}{|l|}{ Tipo de instituição } \\
\hline Feminina & $2(14,3 \%)$ & $12(85,7 \%)$ & \multirow{3}{*}{$p=0,69$} \\
\hline M asculina & $0(0 \%)$ & $1(100 \%)$ & \\
\hline M ista & $9(23,1 \%)$ & $30(76,9 \%)$ & \\
\hline \multicolumn{4}{|l|}{ Número de residentes } \\
\hline 1 a 10 idosos & $2(15,4 \%)$ & $11(84,6 \%)$ & \multirow{3}{*}{$p=0,60$} \\
\hline 11 a 30 idosos & $7(25,9 \%)$ & $20(74,1 \%)$ & \\
\hline Mais de 30 idosos & $2(14,3 \%)$ & $12(85,7 \%)$ & \\
\hline \multicolumn{4}{|c|}{ Presença de consultório odontológico convencional } \\
\hline Sim & $2(66,7 \%)$ & $1(33,3 \%)$ & \multirow{2}{*}{$p=0,10$} \\
\hline Não & $9(17,6 \%)$ & $42(82,4 \%)$ & \\
\hline
\end{tabular}

Tabela 3. Relação entre a oferta de atenção odontológica e a presença de consultório odontológico convencional nas instituições de longa permanência filantrópicas e privadas de Belo H orizonte. Belo Horizonte (M G), 2006.

\begin{tabular}{|c|c|c|c|c|}
\hline \multirow{3}{*}{ Existe atenção odontológica } & \multicolumn{4}{|c|}{ Possui consultório odontológico convencional } \\
\hline & \multicolumn{2}{|c|}{ Filantrópicas } & \multicolumn{2}{|c|}{ Privadas } \\
\hline & Sim & Não & Sim & Não \\
\hline \multirow{3}{*}{$\begin{array}{l}\text { Sim } \\
\text { Não }\end{array}$} & $2(66,7 \%)$ & $1(5 \%)$ & 0 & $8(25,8 \%)$ \\
\hline & $1(33,3 \%)$ & $19(95 \%)$ & 0 & $23(74,2 \%)$ \\
\hline & \multicolumn{2}{|c|}{$p=0,03$} & & \\
\hline
\end{tabular}

dor, o qual retornou após uma semana para recolhêlos, 12 coordenadores deinstituições (19\%) se recusaram a participar. As perdas foram homogêneas entre as regionais e as modalidades das instituições (filantrópicas=7 e privadas=6).

Os resultados deste trabal ho em relação ao número deinstituições e deidosos residentes em
Belo Horizonte foram comparados aos de Chaimowicz e Greco ${ }^{12}$, que avaliaram a dinâmica de institucionalização deidososnessemunicípio, em 1994. Nesse período, o número total de instituições de longa permanência aumentou de 40 para 67. H ouve o surgimento de 31 novas instituições privadas e uma redução de duas instituições fi- 


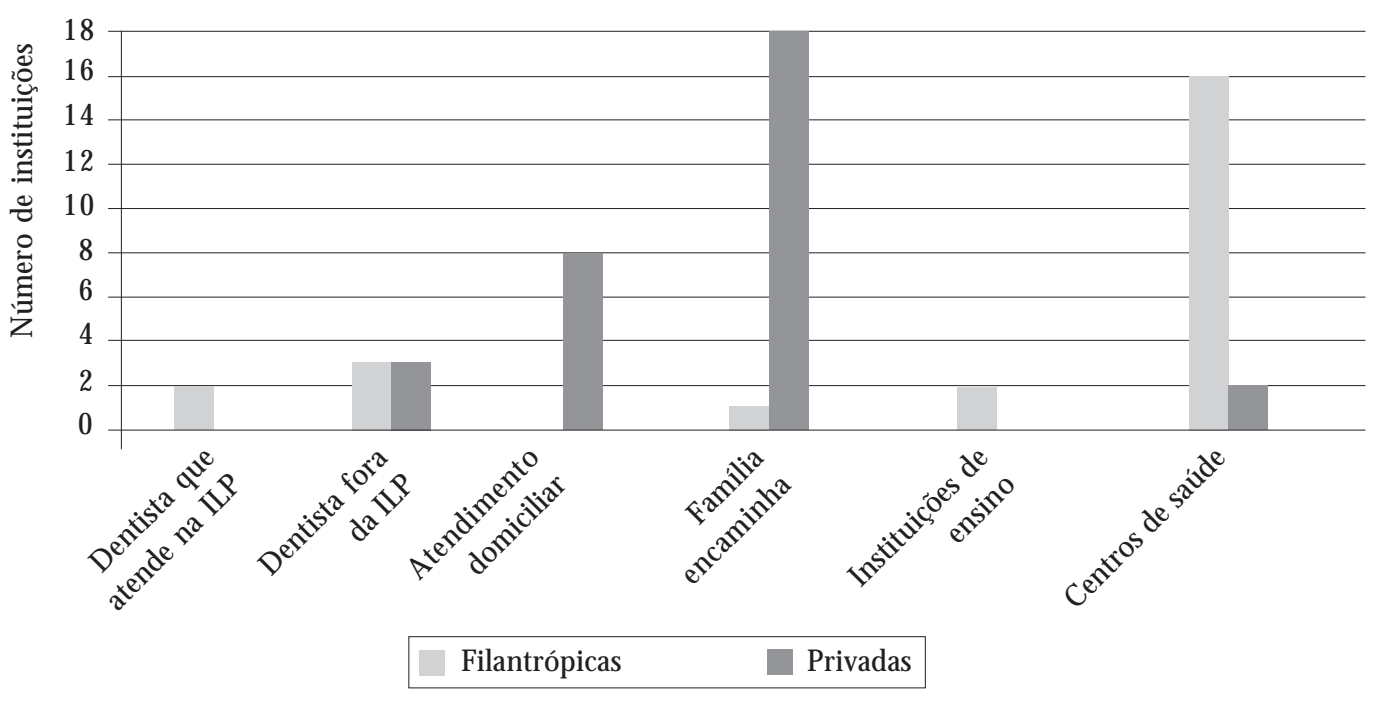

Gráfico 2. Distribuição das instituições de longa permanência filantrópicas e privadas de Belo Horizonte (MG) por regiões administrativas do município.

lantrópicas. Em 1994, as instituições filantrópicas, sem fins lucrativos, vinculadas à Sociedade São Vicente de Paulo ou entidades religiosas, representavam $80 \%$ ( 32 instituições) enelas residiam $85,3 \%$ do total de residentes ( 1.040 idosos), e nas oito instituições privadas residiam apenas 179 idosos. No presente estudo, as instituições filantrópicas mantinham $68 \%$ do total de residentes (1.134 idosos), eas particulares, apesar de representarem a maioria (55\%), abrigavam menor número de idosos (535). Essa mudança re presentou um aumento de $36,9 \%$ no número de idosos institucionalizados (de 1.219 para 1.669), crescimento também observado na população idosa. H ouve um aumento de $39,6 \%$ no número de indivíduos com 60 anos ou mais, de 1991 (146.537 idosos) para 2000 (204.573) (13. $^{13}$.

Vale ressaltar que houve crescimento das instituições privadas. M aior percentual de instituições denatureza privada, com finalidade lucrativa, foi também observado no Rio de Janeiro ${ }^{14}$. Essa mudança no perfil de distribuição das instituições delonga permanência em Belo H orizonte leva a uma reflexão sobre 0 acesso à institucionalização, pois as novas casas são prioritariamente destinadas àqueles que possuem recursos financeiros para custear a sua residência, esobre o papel do poder público e sua responsabilidade diante de uma população crescente de idosos. Apesar de a Política Nacional do Idoso definir que 0 atendimento ao idoso deve ser, preferencialmente, na modalidadenão asilar, determina que a modalidade asilar de assistência social ao idoso ocorre no caso da inexistência do grupo familiar, abandono e carência de recursos financeiros próprios ou da própria família ${ }^{15}$. Entretanto, outros fatores podem determinar a institucionalização. As modificações na estrutura familiar e a dinâmica da sociedade, em que há, cada vez mais, a inserção do maior número possível de integrantes da família no mercado de trabalho, em especial a mulher, expõem a pessoa idosa ao risco de institucional ização ${ }^{16}$. Segundo Perline et al. ${ }^{16}$, em determinadas situações ou períodos, a capacidade da família para o cuidado pode estar comprometida ou fragilizada e, nestas condições, o idoso pode constituir-se num entrave à autonomia dos familiares, seja pelas demandas do cotidiano, que não Ihes possibilita conciliar cuidado e atividades de trabalho e do lar, ou pela impossibilidade de, dentre os familiares, encontrar um ou mais membros que se disponibilizem e se responsabilizem pelo cuidado do idoso. Além disso, a opção da família pelainstitucional ização podefundamentar-senas dificuldades de relacionamentos dos membros da família com o idoso, o adoecimento do idoso gerando dependência para as atividades básicas e instrumentais de vida diária e a dificuldade de se manter um cuidador formal. No entanto, a opção por residir em uma instituição de longa 
permanência pode partir do próprio idoso, buscando atenção, conforto e atendimento às suas necessidades básicas. Segundo Herédia et al. ${ }^{17}$, a ausência de um companheiro é um fator determinante para 0 asilamento.

Como em outros estudos, a maioria dos idosos institucionalizados de Belo Horizonte é do sexo feminino ${ }^{12,14,18,19}$. Essa distribuição se repete na população de idosos como um todo. Em Belo Horizonte, no censo demográfico realizado em 2000, o número absoluto de mulheres com 60 anos ou mais ( 123.959 - 60,6\%) foi maior que 0 de homens na mesma faixa etária (80.614 $39,4 \%)^{13}$. A maior institucionalização das mulheres pode ser explicada também por elas se tornarem viúvas mais cedo, apresentarem maior dificuldade para se casar ou para recasamentos após separação ou viuvez, e menores níveis de instrução, taxa de ocupação e renda ${ }^{12}$. A presença de instituições que aceitam somente mulheres pode contribuir para a diferença observada. De maneira semelhante, M oreno eVeras ${ }^{14}$ observaram que $25 \%$ das instituições delonga permanência no Rio dejaneiro recebiam exclusivamente mulheres.

As instituições privadas e filantrópicas estavam heterogeneamente distribuídas nas noveregionais administrativas de Belo Horizonte, com as privadas mais frequentemente localizadas nas regionais Centro-Sul e Pampulha. Esse achado foi analisado empregando-se o Índice de VulnerabilidadeSocial (IVS), queidentificou as regiões da cidade de Belo H orizonte onde vive a população mais vulnerável à exclusão social; quanto maior 0 índice, pior a situação da população ${ }^{20}$. Asregionais Centro-Sul e Pampulha apresentam os menores valores de IVS do município ${ }^{21}$. Considerando as faixas do IVS, 70,2\% das instituições privadas localizavam-seem regiões com IVS até 0,4 , ao passo que $66,6 \%$ das filantrópicas, em regiões com IV S de 0,4 a 0,63. As instituições privadas, que possuem fins lucrativos, se instalaram preferencialmente em locais onde há maior concentração de indivíduos menos vulneráveis à exclusão social. Um dos aspectos na obtenção do índiceéa renda. Assim, indivíduos que possuem maior renda podem apresentar maior chance de pagar pela sua moradia em uma instituição particular para idosos.

A portarian ${ }^{\circ} 810^{22}$, de 22 de setembro de 1989 , do M inistério da Saúde, descreve as normas e os padrões para funcionamento de casas de repouso, clínicas geriátricas e outras instituições destinadas ao atendimento de idosos. Segundo o documento, os recursos humanos mínimos necessários ao funcionamento das instituições asila- res e suas respectivas cargas horárias são a assistência médica (dez horas semanais), assistência de enfermagem (24 horas por dia), assistência psicossocial (incluídos o assistente social eo psicológico - dez horas semanais), assistência nutricional (cinco horas semanais) e assistência de reabilitação (que incluem o terapeuta ocupacional e o fisioterapeuta - dez horas semanais), incluindo ainda a necessidade de assistência odontológica, farmacêutica, apoio jurídico, administrativo, atividades delazer eserviços gerais. Como no presente estudo, outros autores observaram a inexistência da equipemultidisciplinar nasinstituições ${ }^{18,19}$.

A presença de cirurgião-dentistana equipede profissionais de saúde foi muito pequena; além disso, nenhuma instituição relatou possuir outros profissionais atuando especificamente com saúdebucal, como técnico em higiene dental. Yamamoto e Diogo ${ }^{19}$ observaram, na cidade de Campinas (SP), que duas das seis instituições avaliadas possuíam dentistas no quadro de profissionais de saúde. No entanto, os dirigentes das instituições não souberam informar sobre carga horária etipo de vínculo de trabal ho desses profissionais. N ascimento eSilva et al. ${ }^{18}$ observaram, em uma cidade do interior de São Paulo, que nenhuma das instituições de longa permanência avaliadas, sendo três de caráter privado e três filantrópicas, possuía dentista no corpo técnico administrativo atuante.

Segundo Shinkai e Cury'23, a participação do cirurgião-dentista e do técnico de higiene dental ainda não é muito efetiva no contexto de atenção interdisciplinar ao idoso. A literatura sobre a odontologia na prática interdisciplinar na terceiraidadeéquase inexistente, havendo poucasmenções dedentistas nas equipes deassistência ao idoso. Segundo os autores, uma das razões dessa dificuldade de vivência interdisciplinar para a odontologia reside na formação de base tecnicista, que privilegia o enfoque da superespecialização. É uma visão limitada de postura odontológica intervencionista, que tem suas raízes históricas fincadas em conceitos ultrapassados de saúde bucal desvinculada da saúde geral, sendo totalmente inadequada para o atendimento ao idoso.

A pequena participação do dentista ocorre não por ausência de demandas odontológicas na terceira idade. Ferreira ${ }^{7}$ observou, entre os idosos institucionalizados de Belo Horizonte, alta prevalência de lesões de mucosa bucal, higiene bucal precária, alto índice de cárie dentária e doença periodontal e grande necessidade de próteses dentárias. 
No presente estudo, a atenção odontológica não foi influenciada por modalidade, tipo, localização, tempo de existência e número de idosos residentes nas instituições, sugerindo que, de maneira geral, a odontologia assume pequena prioridade entre as ações de saúde disponibilizadas para o idoso nas instituições. Segundo Colussi e Freitas ${ }^{24}$, a saúde bucal tem sido relegada ao esquecimento quando se discute a saúde da população idosa brasileira. A perda total dos dentes ainda é aceita pela sociedade como algo normal e natural do envelhecimento.

M esmo nas instituições com atenção odontológica, o encaminhamento do idoso foi realizado diante dealguma necessidade odontológica percebida por algum profissional da instituição ou alguma queixa do idoso. Este achado sugerea negligência da instituição quanto ao cuidado bucal, uma vez que não é mencionada a adoção de medidas preventivas ou de cuidados periódicos. Uma ação só é desencadeada quando sinais ou sintomas das doenças bucais se tornam visíveis. $M$ inayo ${ }^{25}$ ressalta que a negligência, conceituada como recusa, omissão ou fracasso por parte do responsável pelo idoso em lhe aportar os cuidados de que necessita, é uma das formas de violência mais presentes no Brasil. Essa negligência pode ser explicada pela falta de conhecimento, por parte dos coordenadores e até mesmo de quem cuida diretamente do idoso, da importância de medidas preventivas e cuidados periódicos na manutenção da saúde bucal do idoso. Fernandes ${ }^{26}$ relata que o mais grave problema relacionado ao funcionamento de instituições asilares é a falta de preparo dos profissionais que atuam no setor. Segundo o autor, essas dificuldades estão presentes tanto no caso dos dirigentes como no corpo de atendentes, sugerindo, como indispensável, a formulação de programas de formação profissional, treinamento e supervisão voltados para esses profissionais. 0 mesmo autor sugere como alternativa viável o trabalho das instituições juntamente às universidades: a universidade teria na população idosa um campo de estágio e de preparação para os profissionais de saúde, e as instituições beneficiar-se-iam com a prestação de serviços profissionais de melhor qualidade.

A busca do tratamento odontológico somente quando o idoso apresenta sinais e/ou sintomas de doenças bucais sugere também que prevalece, entre os coordenadores das instituições e seus cuidadores, a percepção de uma odontologia curativa. Nesse contexto, deve-se discutir ainda a necessidade de eliminação de preconceitos em relação à atuação da odontologia. Embora a atuação odontológica deva ocorrer em todos os níveis, promoção de saúde, prevenção específica e reabilitação, por meio demedidas integradas entre si e no contexto biopsicossocial do indivíduo, a prevenção primária é destacada como estratégia fundamental para a saúde bucal dos idosos ${ }^{23}$. Recomendações da Federal Dentária I nternacional incluem a aplicação de serviços preventivos extensos para os países em desenvolvimento, e quando os recursos forem escassos, a sua distribuição para a prevenção e a educação em lugar dos procedimentos restauradores ${ }^{27}$. Rosa et al. ${ }^{28}$ reforçam esse aspecto, uma vez que salientam a necessidade de políticas nacionais, estaduais e municipais de saúde com destaquena prevenção em idosos para reduzir os níveis de doença, a fim de que a capacidade de atendimento não seja sufocada pelas necessidades de tratamento. Entretanto, o sucesso de programas de prevenção não dependeapenas de ações governamentais específicas. Ettinger ${ }^{29}$, avaliando estudos realizadosnos Estados Unidos durantetrinta anos, verificou que a diminuição progressiva deedentulismo em adultos ocorreu, provavelmente, devido à alteração de valores e atitudes em relação à saúde bucal. Dessa forma, a melhora nas condições de saúde bucal está essencialmenteligada aos valoresa esta atribuídos pela população. Trata-se de retirar da população de terceira idade o estigma de naturalmente doente, ideia que o próprio indivíduo idoso tem desi, para que as necessi dades de saúde sejam percebidas ese tornem reais. Somentea partir daí ocorrem mudanças de atitudes pessoais, que são pré-requisitos para a reivindicação de medidas específicas e sua aceitação.

Os encaminhamentos dos idosos variaram entreas modalidades das instituições, o que pode refletir a falta de um consenso sobre a melhor forma de atendimento para pacientes com dificuldades de locomoção, como os presentes em instituições geriátricas ${ }^{30}$. Além disso, pode estar relacionado também ao perfil socioeconômico dos idosos residentes. M esmo sem um consultório convencional na instituição, no serviço privado foi relatada a contratação de atendimento odontológico domiciliar, com ou sem consultório odontológico portátil. Portanto, 0 atendimento odontológico ofertado pelas instituições privadas é dependente da disponibilidade de re cursos financeiros do residentee, assim, de acesso de uma minoria de idosos que pode custeá-lo - diretamente, quando pagando ao profissional, ou indiretamente, por meio das mensalidades pagas às instituições. Nas instituições filantrópi- 
cas, 0 atendimento público oferecido pelo Sistema Único de Saúde (SUS), através do centro de saúde, foi a escolha da grande maioria. Percebem-se daí diferenças no acesso do residente aos cuidados odontológicos, sendo estes considerados frequentemente como privilégio enão como um direito do idoso institucionalizado.

Os cuidados diários de higiene bucal devem ser sistematizados e adotados como parte da rotina dos cuidados com o idoso e adequados conforme a sua condição funcional e cognitiva ${ }^{31}$. Grande maioria dos coordenadores avaliados re latou a adoção de uma rotina dehigiene bucal nas instituições. Entretanto, a comparação dos relatos mostrou ausência de sistematização das medidas adotadas, sem menção a procedimentos diferenciados segundo a condição cognitiva efuncional do idoso. Ferreira ${ }^{7}$ observou, entre os idosos institucionalizados de Belo Horizonte, alto índice deplaca dentária ehigieneprecária das próteses dentárias e grande percentual de idosos que dependem de outros para o cuidado, incluindo a higiene bucal. Portanto, há necessidade de adaptar e implantar protocolos com medidas sistematizadas de higiene bucal a serem adotadas nas instituições e, em seguida, promover a capacitação dos cuidadores nessa atividade. Estudos na literatura mostram uma negligência das instituições em relação à higiene bucal dos idosos, justificada pela sobrecarga de trabalho, falta de colaboração do idoso, por ser uma prática desagradável epor desconhecimento dos cuidadores ${ }^{10,32,33}$. 0 acúmulo de placa bacteriana devido à higienização bucal inadequada podelevar, em idosos fragilizados, a condições sistêmicas de risco, como má nutrição, pneumonias e doenças cardiovasculares $^{34-36}$. Dessa forma, é evidente que as práticas dehigiene bucal, de baixo custo, têm o potencial de reduzir os custos resultantes de má condição de saúde bucal, levando a uma economia eà promoção de um cuidado de qualidade.

\section{Conclusões}

Com base nos resultados deste trabalho, podese concluir em relação às instituições de longa permanência geriátricas de Belo H orizonte que:

- As instituições filantrópicas existem em maior número, são maisantigas epossuem maior número de residentes que as privadas. $N$ as duas modalidades, há maior número de residentes do sexo feminino. Há uma distribuição desigual das instituições nas regionais administrativas do município.

- Os cirurgiões- dentistas estão entre os profissionais menos frequentes na equipe de saúde dasinstituições. A atenção odontológica foi muito pequena, independentemente da modalidade da instituição, da sua localização, do tipo, do tempo de fundação, da presença de consultório odontológico convencional e do número de idosos residentes.

- A atenção odontológica diferiu entre as modalidades das instituições delonga permanên$\mathrm{cia}$, sendo quenas privadas ocorreo atendimento domiciliar na própria instituição, mesmo sem consultório odontológico convencional, e nas filantrópicas a atenção existe nas instituições que possuem consultório odontológico convencional.

- Há necessidade de adoção de protocolos sistematizados de higiene bucal nas instituições de longa permanência de Belo H orizonte.

\section{Colaboradores}

RC Ferreira elaborou o delineamento do estudo, realizou a análise e a interpretação dos dados e participou da redação do artigo; CW Schwambach coletou os dados e contribuiu na redação do artigo; CS M agalhães e AN M oreira orientaram o delineamento do estudo, acompanharam 0 andamento do trabalho, participaram da análise e discussão dos resultados e contribuíram para a revisão final do artigo.

\section{Agradecimentos}

À promotora de Justiça Simone Montez Pinto $M$ onteiro, à assistente social Sônia e à psicóloga Patrícia, da Promotoria de D efesa da Pessoa Portadora de D eficiência Física e do Idoso. 
1. Ramos LR, Rosa TEC, Oliveira ZM, M edina MCG, Santos FRG. Perfil do idoso em área metropolitana na Região Sudeste do Brasil. Rev Saude Publica 1993; 27(2):87-94.

2. Chaimowicz F. A saúde dos idosos brasileiros às vésperas do século XXI: problemas, projeções e alternativas. Rev Saude Publica 1997; 31(2):184-200.

3. Brasil. M inistério da Saúde. Brasil: Coordenação Nacional de Saúde Bucal. Projeto SB Brasil 2003: condições de saúde bucal da população brasileira 2002-2003 resultados principais. [documento na Internet] [acessado 2006 abr 12]. Disponível em: www.bvsms. saude.gov.br/bvs/publicacoes/projetosb_brasil2004

4. Jorge Júnior J, Almeida OP, Bozzo L, Scully C, Graner E. Oral mucosal health and disease in institutionalized elderly in Brazil. Community Dent Oral Epidemiol 1991; 19(3):173-175.

5. Silva SRC, Valsecki Jr A. Assessment of oral health in an elderly Brazilian population. Rev Panam Salud Publica 2000; 8(4):268-271.

6. Silva DD, Sousa MLR, Wada RS. Saúde bucal em adultos e idosos na cidade de Rio Claro, São Paulo, Brasil. Cad Saude Publica 2004; 20(2):626-631.

7. Ferreira RC. Saúde bucal de idosos residentes em instituições delonga permanência de Belo H orizonte[tese]. Belo Horizonte: Universidade Federal de M inas Gerais; 2007.

8. Kossioni AE, Karkazis HC. Socio-medical condition and oral functional status in an older institutionalized population. Gerodontology 1999; 16(1):21-28.

9. Brasil. Secretaria de Políticas de Assistência Social. Departamento de Desenvolvimento da Política de Assistência Social. Gerência de Atenção à Pessoa Idosa. Portaria SAS-073, de 10 de maio de 2001. Estabelece normas de funcionamento de serviços de atenção ao idoso no Brasil. Diário Oficial da União 2001; 10 maio.

10. Chalmers JM, Levy SM, Buckwalter KC, Ettinger $R L$, Kambhu PP. Factors influencing nurses'aides' provision of oral care for nursing facility residents. Spec Care Dentist 1996; 16(2):71-79.

11. Babbie E. M étodos de pesquisa de survey. 3a ed. Belo H orizonte: UFM G; 1999.

12. Chaimowicz F, Greco DB. Dinâmica da institucionalização de idosos em Belo Horizonte, Brasil. Rev Saude Publica 1999; 33(5):454-460.

13. Instituto Brasileiro de Geografia e Estatística (IBGE). Tábuas completas de mortalidade [documento na Internet]; 2007 dez [acessado 2008 han 15]. Disponível em: http://www.ibge.gov.br/home/presidencia/ noticias/noticia visualiza.php?id noticia $=1043 \& i d$ pagina $=1$

14. Moreno $A B$, Veras R. 0 idoso e as instituições asilares no município do Rio de Janeiro. Gerontologia 1999; 7(4):167-177.
15. Brasil. Lei no 8.842 , de 4 de janeiro de 1994. Dispõe sobre a Política Nacional do Idoso, cria o ConseIho Nacional do Idoso e dá outras providências [legislação na Internet]. 1994 [acessado 2005 jul. 17]. Disponível em: http://www.cfess.org.br/pdf/ legislacao_idoso_8842.pdf

16. Perline N $\bar{M} O G$, Leite MT, Furini AC. Em busca de uma instituição para a pessoa idosa morar: motivos apontados por familiares. Rev Esc Enferm USP 2007; 41(2):229-236.

17. Herédia VBM, Cortelletti IA, Casara MB. Institucionalização do idoso: identidade e realidade. In: Cortelletti IA, Casara M B, Herédia VBM, organizadores. Idoso asilado: um estudo gerontológico. Caxias do Sul: Ed. PUC-RS/Educs; 2004. p. 20-36.

18. Nascimento e Silva EB, Pereira NG, Garcia YR. A instituição e o idoso: um estudo das características da instituição e do perfil de seus moradores. Gerontologia 1998; 6(1):167-176.

19. Yamamoto A, Diogo MJD. Os idosos e as instituições asilares do município de Campinas. Rev Latino-Am Enfermagem 2002; 10(5):660-666.

20. Nahas MI. Experiência de construção e perspectivas de aplicabilidade de índices e indicadores na gestão urbana da qualidade de vida: uma síntese da experiência de Belo H orizonte [artigo na Internet]. 2000 [acessado 2007 jul 8]. Disponível em: http://portal1.pbh.gov.br/pbh/ index.html?id_conteudo $=1747 \&$ id_nivel $1=-1$ \# indicadores

21. Minas Gerais. Prefeitura de Belo Horizonte. Planejamento e desenvolvimento urbano: exclusão social 2000 [documento na Internet]; 2000 [acessado 2007 jul 8]. Disponível em: http://portal1.pbh.gov.br/ pbh/index.html?id_conteudo=1747\&id_nivel 1=l\#indicadores

22. Brasil. Portaria n 810, de 22 de setembro de 1989. A prova as normas e padrões para o funcionamento de casas de repouso, clínicas geriátricas e outras instituições destinadas ao atendimento de idosos. Diário Oficial da União 1989; 22 set.

23. Shinkai RSA, Cury AADB. O papel da Odontologia na equipe interdisciplinar: contribuindo para a atenção integral ao idoso. Cad Saude Publica 2000; 16(4):1099-1109.

24. Colussi CF, Freitas SFT. Aspectos epidemiológicos da saúde bucal do idoso no Brasil. Cad Saude Publica 2002; 18(5):1313-1320.

25. M inayo MCS. Violência contra idosos: relevância para um velho problema. Cad Saude Publica 2003; 19(3):783-791.

26. Fernandes ES. O que pode a cidade fazer pelos seus idosos. M aturidade 1990; 6:11-18.

27. Fédération Dentaire Internacionale (FDI). Necesidades de salud bucal del anciano. FDI Wordh Dental Federation 1993; 2:13-15. 
28. Rosa AGF, Fernandez RAC, Pinto VG, Ramos LR. Condições de saúde bucal em pessoas de 60 anos ou mais no município de São Paulo (Brasil). Rev Saude Publica 1992; 26(3):155-160.

29. Ettinger RL. The unique oral health needs of an aging population. Dent Clin North Am 1997; 41(4):633-649.

30. M acEntee MI. Caring for elderly long-term care patients: oral health related concerns and issues. Dent Clin North Am 2005; 49(2):429-443.

31. Chalmers J, Johnson V. Evidence-based protocol: oral hygiene care for functionally dependent and cognitively impaired older adults. J Gerontol Nurs 2004; 30(11):5-12.

32. Adams R. Qualified nurses lack adequate knowledge related to oral health, resulting in inadequate oral care of patients on medical wards. Journal Advanced Nursing 1996; 24:552-560.

33. Coleman P. Improving oral health care for the frail elderly: a review of widespread problems and best practices. Geriatr N urs 2002; 23(4):189-199.

34. Walls AW, Steele JG, Sheiham A, Marcenes W, M oynihan PJ. Oral health and nutrition in older people. J Public Health Dent 2000; 60(4):304-307.

35. Shay K, Scannapieco FA, Terpenning MS, Smith BJ, Taylor GW. Nosocomial pneumonia and oral health. Spec Care Dentist 2005; 25(4):179-187.

36. Demmer RT, Desvarieux M. Periodontal infections and cardiovascular disease: the heart of the matter. J Am Dent Assoc 2006; 137(Suppl.2):14S-20S.

Artigo apresentado em 08/02/2008

Aprovado em 10/10/2008

Versão final apresentada em 20/11/2008 